\title{
Steroid-resistant nephrotic syndrome as the initial presentation of nail-patella syndrome: a case of a de novo LMX1B mutation
}

Tomohiro Nakata ${ }^{1 \dagger}$, Ryo Ishida ${ }^{1 \dagger}$, Yuu Mihara ${ }^{1}$, Atsuko Fujii ${ }^{1}$, Yoshimoto Inoue ${ }^{2}$, Tetsuro Kusaba' ${ }^{1}$, Tsuyoshi Isojima ${ }^{3}$, Yutaka Harita ${ }^{3}$, Chiaki Kanda², Sachiko Kitanaka ${ }^{3}$ and Keiichi Tamagaki ${ }^{*}$

\begin{abstract}
Background: Nail-patella syndrome (NPS) is an autosomal dominant disorder caused by mutations in the LMX1B gene and is characterized by nail dysplasia, skeletal abnormalities, and nephropathy. We herein report a case of steroid-resistant nephrotic syndrome (SRNS) prior to overt orthopedic symptoms in a patient with NPS.

Case presentation: A 24-year-old woman presented to our hospital with knee pain. She had poorly developed nails, hypoplastic patellas, dislocation of the elbows, and iliac horns in the pelvis. At the age of 7, she developed nephrotic syndrome and was diagnosed with primary focal segmental glomerulosclerosis by renal biopsy. She received long-term corticosteroid therapy with no obvious response. Her clinical course and orthopedic manifestations indicated NPS, and a genetic analysis showed a de novo mutation in the LMX1B gene $(c .819+1 G$ > A). Nephropathy in this case was considered to be associated with NPS. Therefore, we discontinued corticosteroids without the exacerbation of nephrotic syndrome.

Conclusions: Patients with NPS may develop nephrotic syndrome prior to overt orthopedic symptoms and only show non-specific findings in renal biopsy at an early stage of NPS nephropathy. Hereditary nephrotic syndrome, often presenting as childhood-onset SRNS, may also be difficult to diagnose in patients with the following conditions: renal symptoms prior to overt extrarenal symptoms, de novo mutations, and non-specific findings in renal biopsy. Therefore, in the management of SRNS in children, we need to reconsider the possibility of hereditary diseases such as NPS even without a family history.
\end{abstract}

Keywords: Nail-patella syndrome, LMX1B, Steroid-resistant nephrotic syndrome

\section{Background}

Nail-patella syndrome (NPS; OMIM 161200) is a pleiotropic autosomal dominant disorder characterized by nail dysplasia, patellar aplasia or hypoplasia, elbow dysplasia, and iliac horns in the pelvis [1-3]. The causative gene of NPS has been identified as the LIM homeobox transcription factor 1 beta (LMX1B; OMIM 602575) gene, which is located on the long arm of chromosome 9 [4]. The incidence of NPS was previously

\footnotetext{
* Correspondence: tamagaki@koto.kpu-m.ac.jp

${ }^{\dagger}$ Equal contributors

${ }^{1}$ Division of Nephrology, Department of Medicine, Kyoto Prefectural

University of Medicine, 465 Kajii-cho, Kamigyo-ku, Kyoto 602-8566, Japan

Full list of author information is available at the end of the article
}

reported to be approximately 1:50,000, with de novo mutations accounting for $12.5 \%$ of NPS cases $[4,5]$.

Nail anomalies have been detected in $95.1 \%$ of NPS patients, patellar aplasia or hypoplasia in $92.7 \%$, iliac horns in $70 \%-80 \%$, elbow dysplasia in $92.5 \%$, ocular hypertension in $7 \%$, and glaucoma in $10 \%$ [3]. The incidence of renal involvement was found to be $37.5 \%$ in NPS patients, and the mean age when renal involvement was detected was 21.7 years [2]. Nephrotic syndrome is exceptional; most patients present with proteinuria and hematuria [2,6]. Renal failure occurs in between 5 and $14 \%$ of NPS patients [2]. 
We herein report a case of childhood-onset steroidresistant nephrotic syndrome (SRNS) that was diagnosed with NPS based on delayed orthopedic symptoms and a genetic analysis following long-term corticosteroid therapy. We also discuss the need to reconsider hereditary diseases in the management of children with SRNS even without a family history.

\section{Case presentation}

A 24-year-old woman presented to our hospital with pain in her knee. At the age of seven, she developed nephrotic syndrome and was diagnosed with primary focal segmental glomerulosclerosis (FSGS) by renal biopsy (Fig. 1). Nephrotic syndrome gradually worsened without symptoms such as edema. She was administered corticosteroids from the age of 18 years, but did not respond to this therapy. Cyclosporine was then administered, but was discontinued within one week due to the adverse effect of fatigue. Corticosteroids were tapered with proteinuria of 4 to $6 \mathrm{~g}$ per 24 hours.
When she presented to our hospital, she had poorly developed nails and small corneas. Intraocular pressure was normal. She gradually developed pain in her knee over 12 months. X-ray and computed tomography were performed to systemically evaluate the orthopedic morphology, and revealed hypoplastic patellas, dislocation of the elbows, and iliac horns in the pelvis (Fig. 2). Laboratory data showed hypoalbuminemia and the excretion of a large amount of protein in the urine, indicating nephrotic syndrome with renal insufficiency. Other serological results revealed that the secondary causes of nephrotic syndrome such as autoimmune disease, hematological diseases, or infection were negative (Table 1). Her clinical course of SRNS and orthopedic manifestations indicated NPS; therefore, we performed a genetic analysis, which showed a heterozygous mutation in the first base of the fifth intron of the LMX1B gene $($ c. $819+1 G>$ A) (Fig. 3). Since this mutation was not detected in her parents, our case was considered to have a de novo mutation.

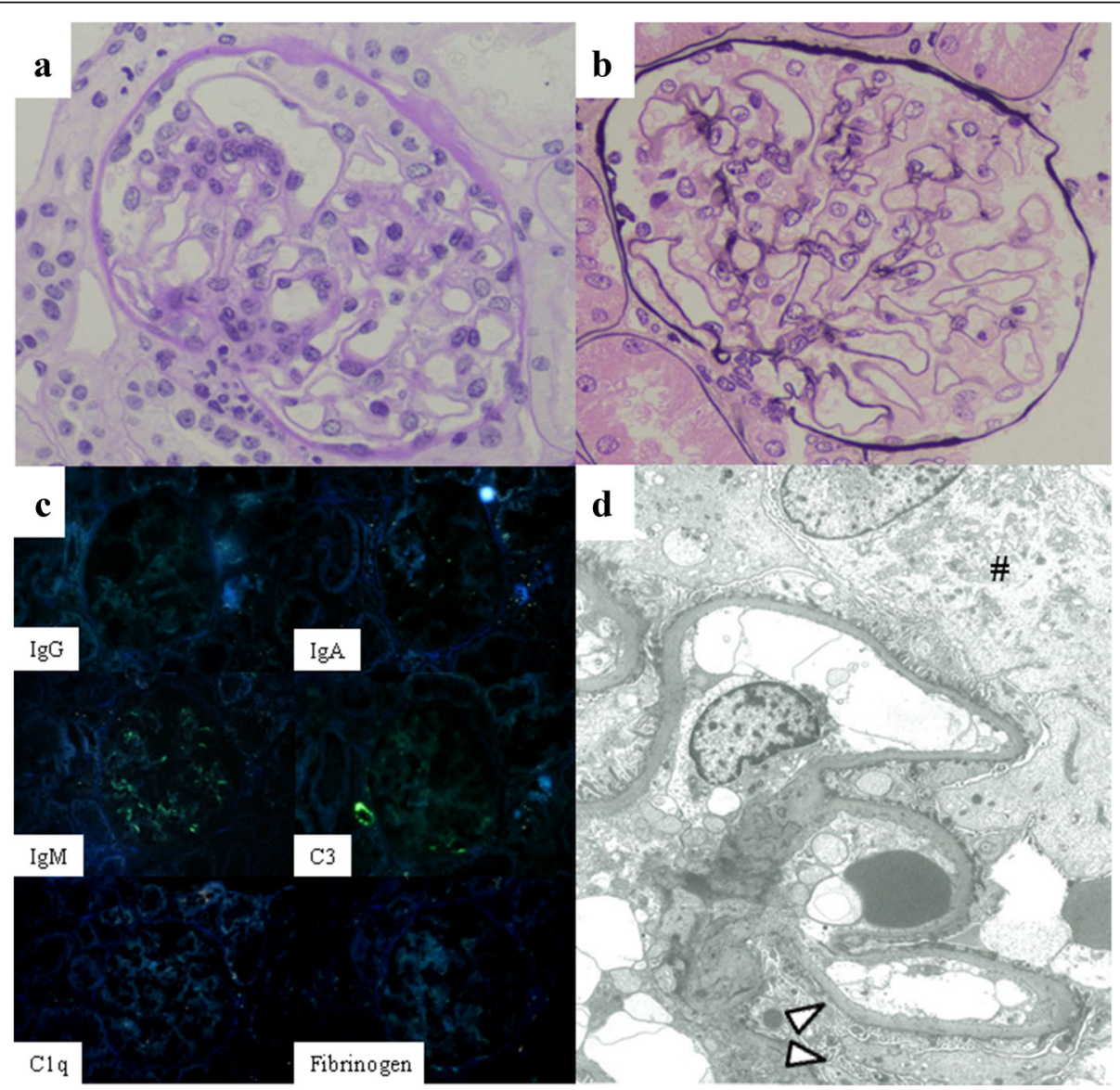

Fig. 1 Renal biopsy at the age of 7. Light microscopy only showed focal glomerular lesions (a) Periodic-Acid Schiff stain x400, (b) Periodic acid-methenamine-silver stain $\times 400$ ). Immunofluorescence showed slightly positive staining for IgM and C3 within glomerular segmental lesions (c). Electron microscopy revealed mild irregular thickening of the GBM and swollen podocytes (\# in $\mathbf{d}$ ) with partial foot process effacement (arrowhead in $\mathbf{d}$ ). Neither electron-lucent areas within the GBM nor electron-dense deposits were detected (d) 

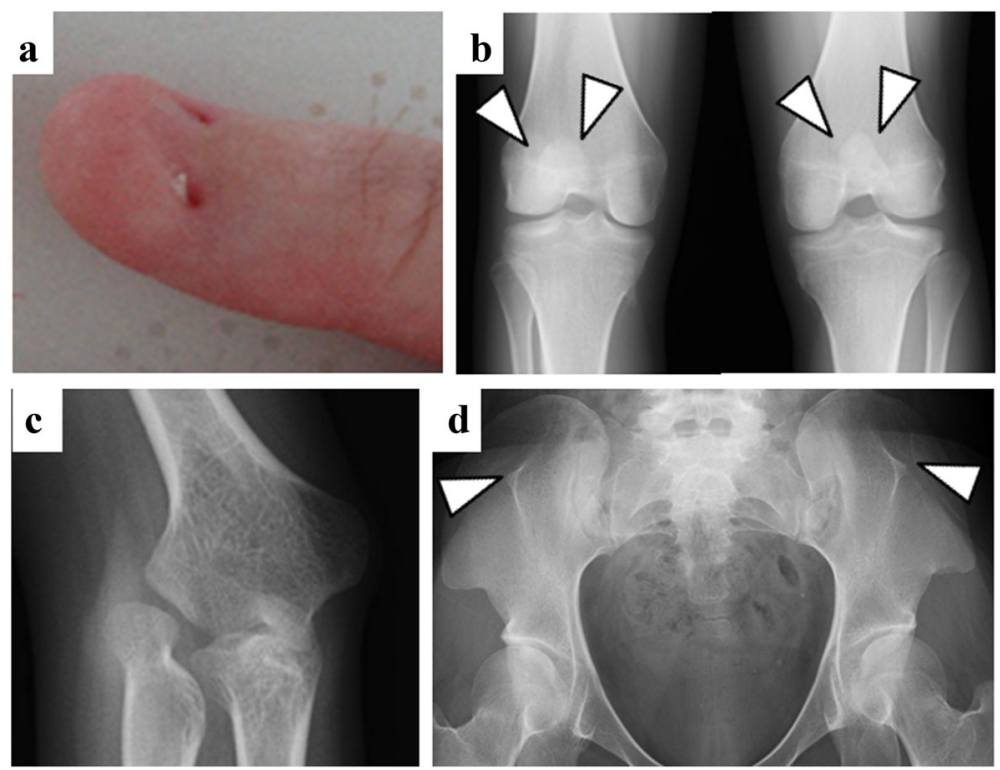

Fig. 2 Nail abnormalities and orthopedic manifestations at the age of 24. She presented with poorly developed nails (a). An x-ray examination revealed hypoplastic patellas (b), dislocation of the elbows (c), and iliac horns in the pelvis (d)

Table 1 Laboratory data on admission

\begin{tabular}{|c|c|c|c|}
\hline Peripheral blood & & Serological tests & \\
\hline WBC & $12100 / \mu \mathrm{l}$ & $\begin{array}{l}\text { Anti-nuclear } \\
\text { antibodies }\end{array}$ & Negative \\
\hline $\mathrm{RBC}$ & $515 \times 10^{4} / \mu \mathrm{l}$ & Rheumatoid factor & Negative \\
\hline $\mathrm{Hb}$ & $14.8 \mathrm{~g} / \mathrm{dl}$ & $\lg G$ & $323 \mathrm{mg} / \mathrm{dl}$ \\
\hline $\mathrm{Ht}$ & $42.9 \%$ & $\lg A$ & $179 \mathrm{mg} / \mathrm{dl}$ \\
\hline Plt & $43.3 \times 10^{4} / \mu \mathrm{l}$ & $\lg M$ & $259 \mathrm{mg} / \mathrm{dl}$ \\
\hline Blood chemistry & & C3 & $80.5 \mathrm{mg} / \mathrm{dl}$ \\
\hline $\mathrm{TP}$ & $4.8 \mathrm{~g} / \mathrm{dl}$ & C4 & $14.6 \mathrm{mg} / \mathrm{dl}$ \\
\hline Alb & $2.8 \mathrm{~g} / \mathrm{dl}$ & $\mathrm{CH} 50$ & $35.0 \mathrm{U} / \mathrm{ml}$ \\
\hline AST & $11 \mathrm{IU} / \mathrm{ml}$ & $\mathrm{Clq}$ & Negative \\
\hline ALT & $14 \mathrm{IU} / \mathrm{ml}$ & HBs antigen & Negative \\
\hline LDH & $240 \mathrm{IU} / \mathrm{ml}$ & Anti-HCV antibody & Negative \\
\hline BUN & $12.1 \mathrm{mg} / \mathrm{dl}$ & Urinalysis & \\
\hline $\mathrm{Cr}$ & $1.01 \mathrm{mg} / \mathrm{dl}$ & Gravity & 1.013 \\
\hline eGFR & $45 \mathrm{ml} / \mathrm{min} / 1.73 \mathrm{~m}^{2}$ & $\mathrm{pH}$ & 6.5 \\
\hline $\mathrm{Na}$ & $140 \mathrm{mEq} / \mathrm{l}$ & Protein & $4+$ \\
\hline K & $3.6 \mathrm{mEq} / \mathrm{l}$ & Occult blood & - \\
\hline $\mathrm{Cl}$ & 105 mEq/l & Sediments & \\
\hline LDL & $123 \mathrm{mg} / \mathrm{dl}$ & RBC & $<1 / \mathrm{HPF}$ \\
\hline TG & $157 \mathrm{mg} / \mathrm{dl}$ & & \\
\hline $\mathrm{HbA1c}$ & $5.2 \%$ & Urinary chemistry & \\
\hline CRP & $0.01 \mathrm{mg} / \mathrm{dl}$ & Protein & $7.67 \mathrm{~g} / \mathrm{gCr}$ \\
\hline
\end{tabular}

In a review of renal biopsy at the age of 7 , light microscopy revealed focal glomerular lesions only (Fig. 1a-b). Immunofluorescence showed slightly positive staining for IgM and C3 within glomerular segmental lesions (Fig. 1c). Electron microscopy displayed mild irregular thickening of the glomerular basement membrane (GBM) and swollen podocytes with partial foot process effacement, whereas distinctive lesions such as electron-lucent areas within the GBM were not detected (Fig. 1d). Although we were unable to perform renal biopsy again because of renal atrophy (parenchymal thinning, an indistinct corticomedullary junction, and longitudinal length of $9 \mathrm{~cm}$ by ultrasonography), nephropathy in this case was considered to be associated with NPS. Therefore, we discontinued corticosteroids gradually without the exacerbation of nephrotic syndrome.

\section{Discussion}

NPS is an autosomal dominant disorder characterized by orthopedic manifestations. In our case, NPS was difficult to diagnose early for the following reasons: 1) nephrotic syndrome developed prior to overt orthopedic symptoms; 2) she had no family history; and 3) renal pathology revealed only non-specific findings in the GBM at an early stage of NPS nephropathy. Based on the presence of orthopedic symptoms, we performed a genetic analysis, detected a de novo mutation in LMX1B without this mutation in her parents, and discontinued unnecessary steroid treatments. 


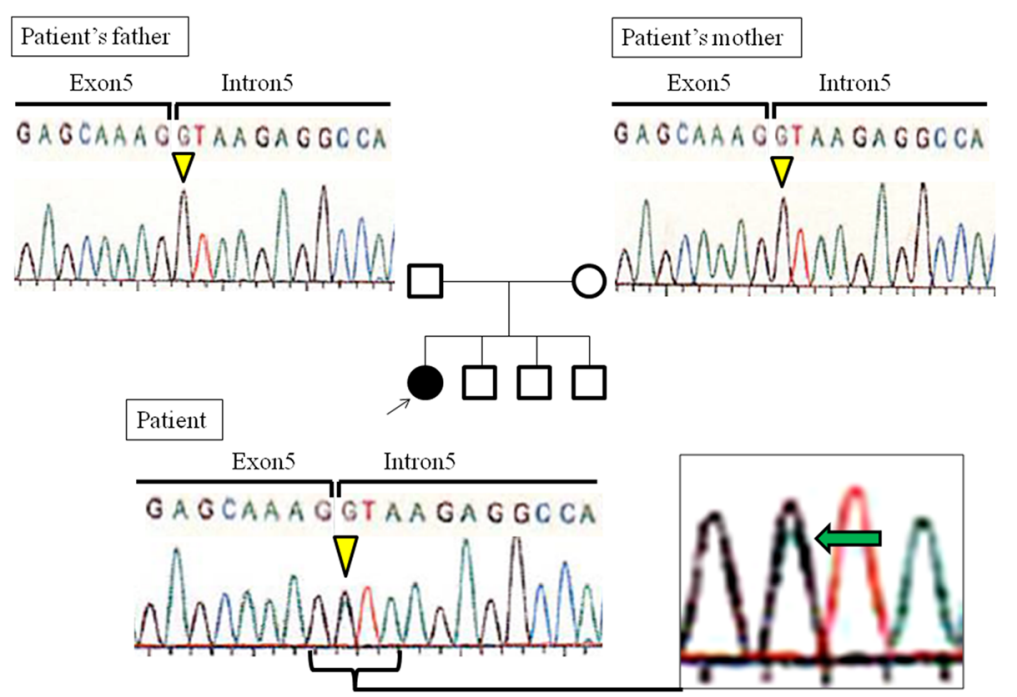

Fig. 3 Genetic analysis. The genetic analysis showed a heterozygous mutation in the first base of the fifth intron (c.819+1G > A) of the LMX1B gene located on the long arm of chromosome 9. The same mutation was not detected in her parents

LMX1B is expressed in the dorsal mesenchyme of developing vertebrate limbs and plays a critical role in the patterning of the dorsal-ventral axis [7]. Once the dorsal-ventral axis is established, LMX1B activates the expression of a number of dorsalizing genes, suppresses the expression of ventralizing genes, and modulates the development of dorsal distal limb structures such as nails and patellas [4]. In addition to limb development, the LMX1B gene is associated with the development of multiple organs such as the brain, spinal cord, eyes, ears, and kidneys $[8,9]$. In our case, this gene mutation was implicated in the impairments observed in the limbs, nails, and eyes as extrarenal symptoms.

The expression of LMX1B in the kidney was initially detected in the region of the S-shaped body, which gives rise to the glomerulus, and subsequently in the visceral epithelium of the glomerulus in experiments on mice [4]. LMX1B binds to an enhancer sequence in intron 1 of the collagen IV $\alpha 4$ chain and regulates the coordinated expression of collagen IV $\alpha 3$ and $\alpha 4$ chains, which are required for normal GBM morphogenesis [10]. A previous study on heterozygous LMX1B knockout mice demonstrated that LMX1B affects increases in glomerular volume as a compensatory response to the loss of nephrons [11]. Other studies on podocyte-specific LMX1B conditional knockout mice demonstrated that LMX1B is required for the initial differentiation of podocytes and development of foot processes [12, 13]. In addition, LMX1B is considered to bind to sites in the putative regulatory regions of the CD2AP and NPHS2 genes and regulate their expression, which is associated with the formation of the slit diaphragm [13].
More than 140 mutations have been detected in LMX1B, and the mutation in our case was previously reported to result in the loss of function of LMX1B due to a dysfunction in exon 6 [14]. Although comparisons of phenotypes with genotypes have not revealed any relationship between disease severity and the type or location of mutations [14], specific missense mutations in LMX1B have recently been identified in cases of nephropathy without extrarenal manifestations $[15,16]$. In the present case, nephrotic syndrome developed at the age of 7 , followed by overt orthopedic symptoms after the growth period. Therefore, our case is clearly distinguishable from previously reported cases of isolated nephropathy caused by LMX1B mutations.

The renal pathological findings of NPS nephropathy vary from minor glomerular abnormalities to FSGS under light microscopy [17], and irregular increases in the thickness of the GBM are observed with the progression of the disease. Immunofluorescence is typically negative or IgM and C3 are slightly positive within glomerular segmental lesions. In addition, electron microscopy shows irregular thickening of the GBM with electron-lucent areas, which is known as the moth-eaten appearance. The deposition of collagen III has been detected in GBM lesions [17]. However, the extent and distribution of GBM lesions vary individually without any relationship with the age of patients or severity of renal symptoms [17]. Thus, non-specific pathological findings may be observed at an early stage of NPS nephropathy. In our case, only focal segmental glomerular lesions and mild irregular thickening of the GBM without electronlucent areas were detected. Consequently, she was not 
diagnosed with NPS at the age of 7, and received immunosuppressive therapy. Renal involvement in NPS is caused by a LMX1B gene mutation, which dysregulates the production of collagen, and corticosteroids have no effect on this gene mutation. Thus, her clinical course with no response to corticosteroids supported the diagnosis of NPS. There are currently no specific treatments for renal involvement in NPS, except for angiotensinconverting enzyme inhibitors, which reduce proteinuria in NPS patients [5]. Therefore, we discontinued corticosteroids without the exacerbation of nephrotic syndrome.

\section{Conclusions}

Patients with NPS may develop nephrotic syndrome prior to overt orthopedic symptoms and only show nonspecific findings in renal biopsy at an early stage of NPS nephropathy. Additionally, hereditary nephrotic syndrome, often presenting as childhood-onset SRNS, may be difficult to diagnose in patients with the following conditions: renal symptoms prior to overt extrarenal symptoms, de novo mutations, and non-specific findings in renal biopsy. Therefore, in the management of SRNS in children, we need to reconsider the possibility of hereditary diseases such as NPS even without a family history.

\section{Abbreviations}

FSGS: Focal segmental glomerulosclerosis; GBM: Glomerular basement membrane; LMX1B: LIM homeobox transcription factor 1 beta; NPS: Nailpatella syndrome; SRNS: Steroid-resistant nephrotic syndrome

\section{Acknowledgements}

We thank to Mr. Mrozek (language editor) for his involvement in drafting the manuscript and revising it critically for important intellectual content. This work was supported by Health and Labour Sciences Research Grants (H26-015) from the Ministry of Health, Labour and Welfare of Japan.

\section{Availability of data and materials}

The datasets supporting the conclusions of this article are included within the article.

\section{Authors' contributions}

TN collected clinical information and drafted the manuscript. RI supported data collection, its interpretation, and writing of the manuscript. YM, AF, YI, $\mathrm{CK}$, and KT are the original physicians of the patient. TI, YH, and SK carried out genetic studies and evaluated the mutation. TK helped to draft the manuscript. KT was responsible for critical revision of the manuscript for important intellectual content. All authors were involved in the revision of the draft manuscript and approved the final content.

\section{Competing interests}

The authors declare that they have no competing interests.

\section{Consent for publication}

Written informed consent was obtained from the patient and her parents before the genetic analysis. Written informed consent was also obtained from them for the publication of this case report and any accompanying images. A copy of the written consent is available for review by the Editor of this journal.

Ethics approval and consent to participate Not applicable.

\section{Publisher's Note}

Springer Nature remains neutral with regard to jurisdictional claims in published maps and institutional affiliations.

\section{Author details}

${ }^{1}$ Division of Nephrology, Department of Medicine, Kyoto Prefectural University of Medicine, 465 Kajii-cho, Kamigyo-ku, Kyoto 602-8566, Japan. ${ }^{2}$ Division of Nephrology, Kyoto Min-iren Chuo Hospital, 16-1 Nishinokyo Kasuga-cho, Nakagyo-ku, Kyoto 604-8453, Japan. ${ }^{3}$ Department of Pediatrics, Graduate School of Medicine, The University of Tokyo, 7-3-1 Hongo, Bunkyo-ku, Tokyo 113-8655, Japan.

Received: 31 May 2016 Accepted: 15 March 2017

Published online: 23 March 2017

References

1. Beals RK, Eckhardt AL. Hereditary onycho-osteodysplasia (Nail-Patella syndrome). A report of nine kindreds. J Bone Joint Surg Am. 1969;51:505-16.

2. Sweeney E, Fryer A, Mountford R, Green A, McIntosh I. Nail patella syndrome: a review of the phenotype aided by developmental biology. J Med Genet. 2003;40:153-62.

3. Bongers EM, Gubler MC, Knoers NV. Nail-patella syndrome. Overview on clinical and molecular findings. Pediatr Nephrol. 2002;17:703-12.

4. Chen $H$, Lun $Y$, Ovchinnikov D, et al. Limb and kidney defects in $L m \times 1 b$ mutant mice suggest an involvement of LMX1B in human nail patella syndrome. Nat Genet. 1998;19:51-5.

5. Lemley KV. Kidney disease in nail-patella syndrome. Pediatr Nephrol. 2009:24:2345-54.

6. Proesmans W, Van Dyck M, Devriendt K. Nail-patella syndrome, infantile nephrotic syndrome: complete remission with antiproteinuric treatment Nephrol Dial Transplant. 2009;24:1335-8.

7. Feenstra JM, Kanaya K, Pira CU, Hoffman SE, Eppey RJ, Oberg KC. Detection of genes regulated by $L m \times 1 b$ during limb dorsalization. Dev Growth Differ. 2012;54:451-62.

8. Bongers EM, Huysmans FT, Levtchenko E, et al. Genotype-phenotype studies in nail-patella syndrome show that LMX1B mutation location is involved in the risk of developing nephropathy. Eur J Hum Genet. 2005;13:935-46.

9. Dai JX, Johnson RL, Ding YQ. Manifold functions of the Nail-Patella Syndrome gene $L m \times 1 b$ in vertebrate development. Dev Growth Differ. 2009;51:241-50

10. Morello R, Zhou G, Dreyer SD, et al. Regulation of glomerular basement membrane collagen expression by LMX1B contributes to renal disease in nail patella syndrome. Nat Genet. 2001;27:205-8.

11. Endele $S$, Klein $S$, Richter $S$, et al. Renal phenotype in heterozygous $L m \times 1 b$ knockout mice (Lmx1b+/-) after unilateral nephrectomy. Transgenic Res. 2007:16:723-9.

12. Suleiman $H$, Heudobler D, Raschta AS, et al. The podocyte-specific inactivation of $\mathrm{Lm} \times 1 \mathrm{~b}, \mathrm{Ldb} 1$ and $\mathrm{E} 2 \mathrm{a}$ yields new insight into a transcriptional network in podocytes. Dev Biol. 2007;304:701-12.

13. Miner JH, Morello R, Andrews KL, et al. Transcriptional induction of slit diaphragm genes by $L m \times 1 b$ is required in podocyte differentiation. J Clin Invest. 2002:109:1065-72.

14. McIntosh I, Dreyer SD, Clough MV, et al. Mutation analysis of LMX1B gene in nail-patella syndrome patients. Am J Hum Genet. 1998;63:1651-8.

15. Isojima T, Harita Y, Furuyama M, et al. LMX1B mutation with residual transcriptional activity as a cause of isolated glomerulopathy. Nephrol Dial Transplant. 2014;29:81-8.

16. Boyer O, Woerner S, Yang F, et al. LMX1B mutations cause hereditary FSGS without extrarenal involvement. J Am Soc Nephrol. 2013;24:1216-22.

17. Laszik Z, Kambham N, Silva S. Heptinstall's pathology of the kidney: thrombotic microangiopathies. 7th ed. Philadelphia: Lippincott Williams \& Wilkins; 2014 